\title{
Drug Utilization Review of Anesthetics in Surgery Department of Teritiary Care Hospitals of Warangal Region
}

\author{
Lakshmi Ravalitha Gajjala*, Mounika Adepu1, Anuradha Dasari', Korada Baikuntha Prusty², Gondi \\ Arun $^{2}$
}

${ }^{1}$ Department of Clinical Pharmacy, Talla Padmavathi College of Pharmacy, Warangal, Telangana, INDIA 2Department of Pharmacology, Talla Padmavathi College of Pharmacy, Warangal, Telangana, INDIA.

\begin{abstract}
Background: The concept of Drug utilization review lends its pivotal role in healthcare system in understanding, interpreting and improving the prescribing, administration and usage of drugs aiming to facilitate their rational use. This implies the prescription of a welldocumented drug in an optimal dose on the right indication within right period of time for right duration. Objective: To assess the use of anesthetics by different anesthesiologists within Warangal region and to evaluate the adopted anesthesia recording procedures. Materials and Methods: The study was a prospective observational study of anesthetic utilization which was carried out for 6 months in the department of Surgery in major tertiary care hospitals of Warangal after approval from institutional ethical committee. The data of patients who underwent surgery were collected in predesigned data collection form and were analyzed for drug utilization review and statistics were drawn descriptively. Results: 300 subjects who have undergone surgeries were enrolled for the study of these maximum of subjects being Adults (54\%) within age group of 20-49. Of all planned surgeries (93\%) and emergency surgeries (7\%) were carried out and classified according to American Society of Anesthesiologists (ASA) grading. Local Anesthesia of which Spinal (78\%) and Blocks (8\%) was employed in major surgeries and General Anesthesia (14\%) in few. Among General anesthetics, Propofol (P), Ketamine (K), Fentanyl (F), Midazolam (M), Suxinyl choline (SC), Vecuronium (V), Neostigmine (N), Thiopental (T) were used in combinations along with pre-anesthetic Glycopyrrolate (G). Combinations include GKV (7\%), GFP (2\%), GVN (2\%), PKFM (3\%) and VFTS (1\%). Among Local Anesthestics, Bupivacaine (B), Lignocaine (L), Xylocaine (X) were used in combination or single. B $(62 \%), B L(9 \%), X(8 \%)$ and B with Bupregesic (BPG) of $(6 \%)$. Majorly, Open Reduction Internal Fixation with plates and screws (31), Open Reduction Internal Fixation with Dynamic Hip Screw (26), Lower Segment Cesarian Section (25), Urethroscopic Lithotripsy + Double J stenting (25), Hysterectomy (22), Hernioplasty (15) surgeries were carried out and many other surgeries of less frequency. Ceftriaxone $(51 \%)$, Ranitidine (80\%), Ketorolac (35\%), Ondansetron (56\%) were majorly prescribed post-operative drugs. Metronidazole (6\%), Tetanus (6\%), Amikacin (22\%) were also prescribed additionally in few surgeries as post-operative drugs. Conclusion: Bupivicaine was most commonly prescribed for spinal. Propofol is most commonly prescribed drug for induction of anaesthesia followed by as maintenance of anaesthesia. Choices were contemplated rational as safety, efficacy and patient satisfaction were considered and outcomes were reliably good.
\end{abstract}

Key words: Anesthesia, Utilization review, ASA grading, Warangal.

\section{INTRODUCTION}

The term anesthesia defines loss of all modalities of sensation, particularly pain, along with reversible loss of consciousness. ${ }^{1}$
Anesthetic is a state which is considered to be the collection of "component" changes in behavior or perception. The components of
DOI: 10.5530/ijopp.12.1.4

Address for correspondence: Dr. Lakshmi Ravalitha Gajjala Pharm. D,

Department of Clinical Pharmacy, Talla Padmavathi College of Pharmacy, Warangal, Telangana, INDIA.

Phone no: +918519978910 Email Id: Ravalitha525@gmail. com

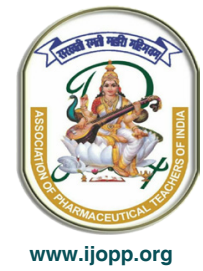


anesthetic state include amnesia, immobility in response to noxious stimuli, attenuation of autonomic responses to noxious stimuli, analgesia and unconsciousness. ${ }^{2}$

American Society of Anesthesiologists classified anesthesia into three main categories, each having many forms and uses.

General Anesthesia, are CNS depressants that are used to bring about a reversible loss of pain sensation and consciousness. Their use permits painful surgical procedures to be performed without the patient being aware of it, or even reacting reflexes. There are number of general anesthetic drugs - some are gases or vapors inhaled through a breathing mask or tube and others include medications introduced through a vein.

Regional Anesthesia- Administration of an injection near to a cluster of nerves to numb the area of body that requires surgery. Receiver remain awake or may be given a sedative, either of the way making no feel of actual surgery taking place. There are several kinds of regional anesthesia; the two most common are spinal anesthesia and epidural anesthesia.

Local Anesthesia- Drug is usually injected into the tissue to numb just the specific location of the body requiring minor surgery. ${ }^{2}$ Local anesthetics are drugs that cause a loss of sensation in a limited area of the body. Unlike general anesthetics, these drugs do not cause unconsciousness. Local anesthetics may be defined as drugs which reversibly block nerve conduction beyond the point of application, when applied locally in an appropriate concentration. ${ }^{3}$

Mechanisms of General Anesthesia produce a progressive depression of the CNS. The degree of depression depends on the concentration of the drug in CNS, which in turn depends on the dynamic balance between the rates at which the gas or vapour is taken up from alveoli by the blood, transported to the blood brain barrier and redistributed by the blood and eliminated by the lungs. An important site of action of general anesthetics is reticular formation in the brainstem. They preferentially depress the ascending Reticular Activating System (RAS) which is concerned in maintaining alertness and wakefulness. By reducing the number of impulses transmitted from this area of the brainstem to the cerebral cortex, these drugs progressively reduces sensory awareness, when a sufficient concentration of the anesthetic is reached, consciousness is lost. Dorsal horn cells of the spinal cord are quite sensitive to general anesthetics, resulting in an interruption of incoming pain impulses.
Mechanism of Local Anesthesia include the main site of action of local anesthetics is cell membrane. The local anesthetics in unionized form easily penetrate through nerve sheath and axon membrane. Within the the axoplasm, the molecules become ionized and block the voltage gated sodium channels. Action of LA is $\mathrm{pH}$ dependent and the penetrability of LA is increased at alkaline $\mathrm{pH}$. Penetrability is very poor at acidic $\mathrm{pH}$ to tissues. In infected tissues there is a low $\mathrm{pH}$ which causes ionization of drugs as such reduces penetration through the cell membranes thus decreases the effectiveness of LA. LA first block small nerve fibers and larger nerve fibers and myelinated are blocked first then the non myelinated of same diameter, sensory are blocked first then the motor fibers because of their high firing rate and longer duration of action potential, fibers at centre are blocked later than the one's located at the circumference of nerve bundle. ${ }^{4}$

The main objectives which are sought for in anesthesia is devoted to a broad generalization about anesthesia for surgery in which the major goals are reduced to two in number-insensibility and immobility. The latter portion deals with objective measurements as a basis for rational activity. Considering these two goals-insensibility and immobility (or relaxation)-as the only essential or desirable departures from the anaesthetized state which need to be produced. All other changes may usually be considered as undesirable. The skeletal muscle relaxants are important in this connection because with them one can distinguish clearly between these two goals and can achieve them separately. The introduction of the skeletal muscle relaxants into anesthesia has brought about an unquestionable improvement in the use of the general anesthetic agents namely a reduction in the concentration of the agents which must be used. ${ }^{5}$

ASA Physical Status Classification -The American Society of Anesthesiologists (ASA) physical status classification system was developed to offer clinicians a simple categorization of a patient's physiological status that can be helpful in predicting operative risk. The ASAPS originated in 1941 and has seen some revisions since that time. ASAPS classification system implicitly assumes that age is unrelated to physiological fitness, an assumption which is simply not true since neonates and the very elderly, even in the absence of disease, are far more "fragile" in their tolerance of anesthetics compared to young adults. However, despite these and other well-known limitations, the ASAPS classification is used ubiquitously (although sometimes uncritically) in providing a convenient description of a surgical patient's overall condition. 
ASA 1: A normal healthy patient. Example: Fit, nonobese (BMI under 30), a nonsmoking patient with good exercise tolerance.

ASA 2: A patient with a mild systemic disease. Example: Patient with no functional limitations and a wellcontrolled disease (e.g., treated hypertension, obesity with BMI under 35, frequent social drinker or is a cigarette smoker).

ASA 3: A patient with a severe systemic disease that is not life-threatening. Example: Patient with some functional limitation as a result of disease (e.g., poorly treated hypertension or diabetes, morbid obesity, chronic renal failure, a bronchospasmtic disease with intermittent exacerbation, stable angina, implanted pacemaker).

ASA 4: A patient with a severe systemic disease that is a constant threat to life. Example: Patient with functional limitation from severe, life-threatening disease (e.g., unstable angina, poorly controlled COPD, symptomatic $\mathrm{CHF}$, recent (less than three months ago) myocardial infarction or stroke.

ASA 5: A moribund patient who is not expected to survive without the operation. The patient is not expected to survive beyond the next $24 \mathrm{~h}$ without surgery. Examples: ruptured abdominal aortic aneurysm, massive trauma and extensive intracranial hemorrhage with mass effect.

ASA 6: A brain-dead patient whose organs are being removed with the intention of transplanting them into another patient.

The addition of "E" to the ASAPS (e.g., ASA 2E) denotes an emergency surgical procedure. The ASA defines an emergency as existing "when the delay in treatment of the patient would lead to a significant increase in the threat to life or body part."

The World Health Organization (WHO) of 1977 defined drug utilization as the marketing, distribution, prescription and use of drugs in a society, with special emphasis on the resulting medical, social and economic consequences. ${ }^{6}$ Pharmacists by virtue of their expertise and their mission of ensuring optimal patient outcomes, should work in the process of medicine use improvement through drug utilization. ${ }^{7}$

\section{MATERIALS AND METHODS}

A six month prospective observational study was carried out in Surgery department of affiliated tertiary care hospitals which are associated with College in Warangal city. A total of 300 cases were collected in which anesthesia usage was determined. The patients of either gender attending surgery were included and out patients of study sites were excluded.

All the relevant and necessary data was collected from manual anaesthetic record chart, review chart and patient drug chart and documented in suitable designed data collection form.

In this study, type of anesthetics mostly administered to the patients whether single or in combination was evaluated. The gender, age of the patient, ASA grading, type of anesthetic technique and type of surgery performed were studied along with post-operative medication preferences. The results were analyzed and evaluated.

\section{RESULTS}

The study entailed 300 subjects, undergoing surgeries with suitable anesthetic administration consisting of $157(52 \%)$ males and 143 (48\%) females. Maximum surgeries are carried out in adults of age group 13-59 $(72 \%)$ followed by geriatrics of age group 60 and above $(26 \%)$ and minimum in pediatrics of age group 1-12 (2\%) at the study sites.

Among all the surgeries carried out 278 (93\%) were planned prior and $22(7 \%)$ surgeries were carried out in emergency conditions. Studying the American society of anesthesiologists (ASA) grading, our study includes 121 $(41 \%)$ grade I, $108(36 \%)$ of grade II, $47(16 \%)$ of grade III, grade $1 \mathrm{E} 14(4 \%)$ and $8(3 \%)$ of grade IIE patients attending for surgeries.

Of all subjects bone fracture was diagnosed in large (85) of 300, followed by Ureteric calculi (34), Renal calculi (25), Patients attending for LSCS were (25), Hernia (14), Ovarian cyst (11) and patients diagnosed with appendicitis are (9). Many other diagnosis were identified in study but are of less frequency as represented in Figure 1 below.

Surgeries carried under various departments were noted of which Orthopedics are of (102), Nephrology (84), Gynaecology (57), General Medicine (46), Gaastroentrology of (6), Oncology of (3) and Cardiology are of (2).

Of all majorly, ORIF with plates and screws (31), ORIF with DHS (26), LSCS (25), URSL+ DJ stenting (25), Hysterectomy (22), Hernioplasty (15) surgeries were done and many other surgeries were also carried out at 
85

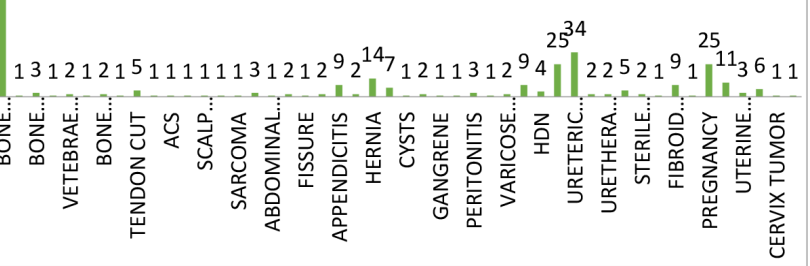

Figure 1: Diagnosis wise distribution of patients.

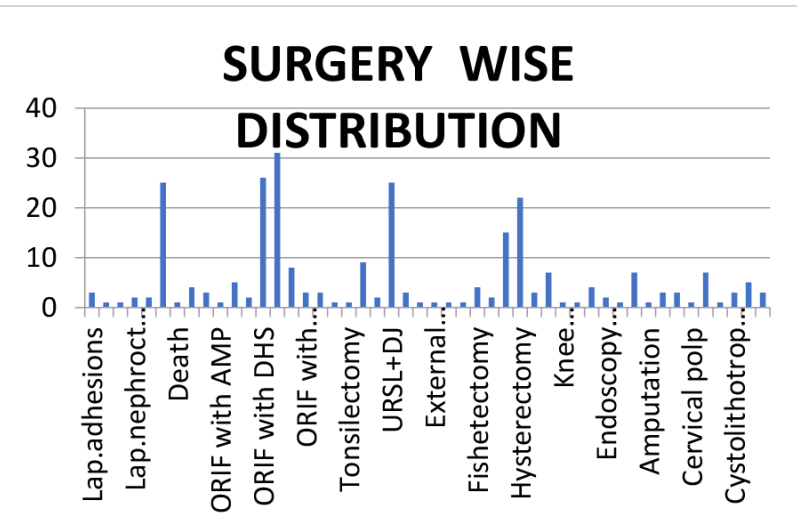

Figure 2: Surgery wise distribution of patients.

tertiary care hospitals but are of less frequency as shown in Figure 2.

The type of anesthesia adopted encompasses General, Regional and Local anesthesia. Of Regional anesthesia majorly observed techniques was spinal and nerve blocks of Local as listed in Table 1.

The frequency of anesthetic drug uses were noted in study as listed in Table 2. The most common LA was found to be Bupivacaine 186 (62\%) and GA anesthetic combination was found to be GKV 22 (7\%).

Most commonly prescribed post-operative antibiotics were ceftriaxone $(59 \%)$, pipercillin + tazobactum $(15 \%)$, ceftriaxone + salbactum $(10 \%)$, cefotaxime + salbactum $(9 \%)$ and aminoglycoside antibiotics were also prescribed such as amikacin $(22 \%)$, gentamycin (3\%). Prophylactic antibiotic tetanus $(6 \%)$ was also prescribed by few physicians post-surgery.

The majorly prescribed proton pump inhibitors Table 1: Frequency of anesthesia types.

\begin{tabular}{ccc} 
TYPE OF ANESTHESIA & $\begin{array}{c}\text { NO OF } \\
\text { PATIENTS }\end{array}$ & $\begin{array}{c}\text { PERCENTAGE } \\
\text { (\%) }\end{array}$ \\
\hline General Anesthesia & 42 & $14 \%$ \\
Regional Anesthesia- Spinal & 233 & $78 \%$ \\
Local Anesthesia - Blocks & 25 & $8 \%$ \\
\hline
\end{tabular}

\begin{tabular}{|c|c|c|c|}
\hline SNO. & ANESTHETIC DRUGS & $\begin{array}{c}\text { NO OF } \\
\text { PATIENTS }\end{array}$ & $\begin{array}{c}\text { PERCENTAGE } \\
(\%)\end{array}$ \\
\hline 1 & BUPIVACAINE(B) & 186 & $62 \%$ \\
\hline 2 & $(B)+$ BUPRIGESIC (BPG) & 19 & $6 \%$ \\
\hline 3 & (B) + LIGNOCAINE (L) & 27 & $9 \%$ \\
\hline 4 & XYLOCAINE (X) & 23 & $8 \%$ \\
\hline 5 & $\begin{array}{c}\text { GLYCOPYRROLATE (G) + } \\
\text { KETAMINE }(\mathrm{K})+ \\
\text { VECURONIUM (V) }\end{array}$ & 22 & $7 \%$ \\
\hline 6 & $\begin{array}{l}\text { (G) + FENTANYL (F)+ } \\
\text { PROPOFOL (P) }\end{array}$ & 05 & $2 \%$ \\
\hline 7 & $\begin{array}{c}(\mathrm{G})+(\mathrm{V})+ \\
\text { NEOSTIGMINE }(\mathrm{N})\end{array}$ & 05 & $2 \%$ \\
\hline 8 & $\begin{array}{c}(P)+(K)+(F)+ \\
\text { MIDAZOLAM }(M)\end{array}$ & 09 & $3 \%$ \\
\hline 9 & $\begin{array}{c}(\mathrm{V})+(\mathrm{F})+ \\
\text { THIOPENTAL }(\mathrm{T})+ \\
\text { SUXINYL CHOLINE (SC) }\end{array}$ & 04 & $1 \%$ \\
\hline
\end{tabular}

was ranitidine $(80 \%)$ and pantoprazole $(18 \%)$ was comparatively less prescribed according to our study. Analgesic drugs were prescribed to manage the pain of which commonly used drugs according to our study included tramadol (43\%), ketorolac $(35 \%)$, diclofenac (4\%). Ondansetron (56\%) was most commonly used antiemetic. Study also resulted in usage of metronidazole $(6 \%)$ an anti-amoebic agent which was prescribed by few physicians post-surgery.

\section{DISCUSSION}

Drug Utilization Review (DUR) is a system of ongoing, systematic, criteria-based evaluation of drug use that ensures that drugs are used appropriately (at the individual patient level). If therapy is deemed to be inappropriate, interventions with providers or patients will be necessary to optimize drug therapy. A DUE is drug- or diseasespecific and can be structured so assessing the actual process of prescribing, dispensing or administering a drug (indications, dose, drug interactions, etc.) is possible. ${ }^{8}$ The concept of prudent drug utilization review facilitates in improved drug use and its application including rational decisions in accordance with condition of patients.

In the present study, use of various agents for anesthesia under few anesthesiologists was observed. The drugs predominantly employed for General, Regional and local anesthesia was noted.

According to our study the type of anesthesia mostly employed was Local anesthesia, of which most followed technique was spinal $(78 \%)$ and blocks of $(8 \%)$ and general anesthesia was employed among (14\%) of subjects.

Indian Journal of Pharmacy Practice, Vol 12, Issue 1, Jan-Mar, 2019 
Our study revealed the maximum use of bupivicaine $(62 \%)$ a widely used local anesthetic agent most commonly used in spinal technique which was potent and has a long duration of action over other drugs. It produces more sensory than motor blockade, was also given in combination with buprigesic $(6 \%)$, other anesthetic agents usage was noted as fallows -xylocaine $(8 \%)$ and combination of bupivucaine + lignocaine $(9 \%)$ in blocks. In correlation with other studies ${ }^{9}$ it was found that safe anesthesia has continued to be administered with the use of xylocaine for most surgical procedures.

General anesthsesia involves usage of many combinational drugs along with pre anesthetics for providing desired therapeutical responses as of Glycopyrolate + Ketamine + Vecuronium (GKV) $(7 \%)$ the paramount combination given to subjects involved in the study, other combinations were Glycopyrolate+ Fentanyl+ Propofol (GFP) (2\%); Glycopyrolte + Vecuronium + neostigmine $(\mathrm{GVN})(2 \%)$; Vecuronium + Fentanyl + Thiopentol + Suxinyl choline (VFTS) of (1\%) employed.

Propofol +Ketamine +Fentanyl + Midazolam (PKFM) $(3 \%)$ was also a combination adopted, IV midazolam was commonly used as an anxiolytic agent. Midazolam has an additive effect with intravenous anaesthetics (propofol, fentanyl) and could prolong the sedative effects after surgery.

Propofol was also employed for maintenance of anaesthesia in short duration surgery as it is associated with rapid, smooth recovery and also not associated with post-operative nausea vomiting

In our study, induction of anaesthesia was done by propofol and maintenance of anesthesia with propofol infusion was well-tolerated by subjects. Other studies also found satisfactory anaesthesia, with good recovery characteristics and a low incidence of postoperative nausea and vomiting with propofol. ${ }^{10}$ Propofol is preferred for the induction and maintenance of general anaesthesia due to low incidences of PONV as suggested according to other studies. ${ }^{9}, 11-15$

Therefore, emetogenic effect of volatile anaesthetics, rather than only the antiemetic effect of Propofol led to the suggestion of prophylactic use of antiemtics post-surgery. This was particularly relevant for surgeries related to gastroenterology. Propofol is associated with a lower risk of postoperative nausea and vomiting, thus providing better patient's satisfaction which was co related with other studies.
Inj. fentanyl was the most commonly used opioid analgesic agent with dose range of $1-2 \mathrm{mcg} / \mathrm{kg}$, repeated intraoperatively especially in procedures of long duration. These findings were similar to previous studies where fentanyl was the most commonly used opioid analgesic. ${ }^{10}$

Ketamine is a dissociative short acting IV anaesthetic agent used for the induction of general anaesthesia. It is used to produce sedation, amnesia, analgesia and to reduce amount of ansthesia post anesthetic complications. It is an ideal induction agent for patients who are hemodynamically unstable as a study resulted ${ }^{16}$ which was in correlation with our study.

The current study included 300 patients who underwent different surgeries, the age wise characterization of the subjects resulted in maximum adults of age group 20-49 with $49 \%$ and minimum surgeries were carried out within age group of 1-12 under pediatrics

In our study males have underwent utmost number of surgeries of $157(52 \%)$ compared with females of $143(48 \%)$.

Of all patients bone fracture was diagnosed in large (85) of 300 , followed by ureteric calculi (34), renal calculi (25), patients attending for LSCS were (25), hernia (14), ovarian cyst (11) and patients diagnosed with appendicitis are 9. Many other diagnoses were also involved in study but are of less frequency.

Of all majorly, ORIF with plates and screws (31), ORIF with DHS (26), LSCS (25), URSL+ DJ Stenting (25), Hysterectomy (22), Hernioplasty (15) surgeries were carried out and many other surgeries were also carried out at teritiary care hospitals but of less frequency.

Among all surgeries carried out patients underwent maximum surgeries under ortho department (102) and minimum under cardiology department (2). Of 300 subjects involved in study few subjects were found to be allergic to NSAIDs (6\%), allergic to iron supplements $(2 \%)$ and a subject was found allergic to ofloxacin + ciprofloxacin prior to surgery.

Most commonly prescribed post-operative antibiotics were ceftriaxone $(59 \%)$ and least was Cefotaxime + Salbactum $(9 \%)$ and aminoglycoside antibiotics were also prescribed such as amikacin (22\%), gentamycin $(3 \%)$. Prophylactic antibiotic tetanus $(6 \%)$ was also prescribed by few physicians post-surgery.

Proton pump inhibitors (PPI's) were prescribed of which ranitidine was maximum and pantoprazole was minimum. 
PPI was routinely prescribed after the surgery to decrease the volume of gastric acid and the $p H$ and also to prevent aspiration. The major prescribed PPI was ranitidine $(80 \%)$ and pantoprazole (18\%) was comparatively less prescribed according to our study.

Analgesic drugs were prescribed to manage the pain post-surgery. The commonly used drugs according to our study included Tramadol (43\%), Ketorolac (35\%), Diclofenac (4\%).

Ondansetron $(56 \%)$ was most commonly used antiemetic. A study shows that antiseratonins (Ondansetron, Granisetron, Ramosetron) are more effective in prophylaxix of PONV than traditional antiemetics (metoclopramide, droperidol). A meta-analysis study shows that ramosetron is superior to ondansetron for preventing PONV ${ }^{10}$ which was also in correlation with our study results. Study also resulted in usage of metronidazole $(6 \%)$ an anti-amoebic agent which was prescribed by few physicians post-surgery.

A satisfactory correlation of 0.79 was established between anesthesia duration and surgery duration indicating the duration of anesthesia and surgery session are correspondingly rational as of protocol guidelines. ${ }^{17}$

\section{Future Directions}

Special interviews with questionnaire filling up session by various anesthesiologists could be done in assessing the common usage patterns of anesthetics and their choices of preferences. Establishment of therapeutic protocols, assessment of adverse effects of anesthetics post surgeries experienced by subjects with ongoing follow-up would help in discerning and assisting anesthesiologists in reducing the frequency of anesthetic related problems.

\section{CONCLUSION}

It was evident from the observation that the choices of anesthesia as well as outcome of procedures were rational and satisfactory from practical understanding. The theoretical understanding therefore holds well in practice of general, regional and local anesthesia at our particular anesthetist units of Warangal.

Our study concluded that spinal anesthesia was mostly adopted by anesthesiologists and Bupivacaine was most common anesthetic drug of choice. Propofol is most commonly prescribed drug for induction and also as maintenance anesthesia in few surgeries.

Finally, conclusion was drawn such that safety, efficacy and patient satisfaction are the hallmarks of good anesthetic care in achieving treatment related goals and choices of anesthesiologists of Warangal were found to be authentically fulfilling them.

\section{ACKNOWLEDGEMENT}

Management of Talla Padmavathi College of Pharmacy and affiliated hospitals, Warangal. Approved by Institutional Human Ethical committee (IEC).

\section{CONFLICT OF INTEREST}

The authors declare no competing interest.

\section{ABBREVIATIONS}

ASA: American Society of Anesthesiologists; ASAPS: American Society of Anesthesiologists Physical Classification; CNS: Central Nervous System; GA: General Anesthesia; LA: Local Anesthesia; DUR: Drug Utilization Review.

\section{REFRENCES}

1. Dr. Suresh B. A text of Pharmacology; 2004 2005, $7^{\text {th }}$ edition. 19.

2. Hardman GJ, Lee E. Limbird, Alfred Goodman Gilman. The Pharmacologic Basis of Therapeutics; $10^{\text {th }}$ edition. 2001;337.

3. Barar FSK. Essentials of Pharmacotherapeutics. $7^{\text {th }}$ edition. 2015.

4. Tara VS, Smita S. Pharmacology For Medical Graduates $2^{\text {nd }}$ Edition. 2013

5. Frumin MJMD. General Anesthesia: Objectives and objectivity A critique. 1957;49(4):219.

6. WHO Expert Committee. The Selection of Essential Drugs, Technical Report Series no. 615. Geneva: World Health Organization. 1977.

7. Folke S, Donald B. Introduction to Drug Utilization Research- Drug Utilization. 2003. ISBN 924 156234X.Ch. 10; 83-4.

8. Drug utilization review. http://apps.who.int/medicinedocs/en/d/Js4882e/8.5.html

9. Olasinde A, Oluwadiya K. Anaesthesia Practice in A Hospital Developing Countries: An 18 months Experience. The Internet Journal of Third World Medicine. 2004;3(1):1-4.

10. Gomathi G, Ghanshyam Y, Pandey BL. Drug utilization review of general anaesthetic agents in a tertiary care hospital. International Journal of Basic and Clinical Pharmacology. 2018;7(3):439-45. DOI: http://dx.doi. org/10.18203/2319-2003.ijbcp20180656

11. Marsha M, Cohen MM, Mhsc F, Peter GD, Frcpc M. A Survey of 112,000 Anaesthetics At One Teaching Hospital (1975-83). Can Anaesth Soc J. 1986;33(1):22-31.

12. Gavin M, Md Peter A. Study of Anesthetic Drug Utilization In Different Age Groups. Journal of Clinical Anesthesia. 2003;15(3):194-200. (c) 2003 Elsevier Science Inc.

13. Amol EP, Yashashri CS, Snehalata VG, Sweta VS. Drug Utilization and Off-Label Use of Medications in Anaesthesia in Surgical Wards of a Teaching Hospital. DOI: 10.4103/0019-5049.170032

14. Helena G. Departamento De Investigação E Desenvolvimento, Bial, Porto Drug Utilization Studies. Arquivos De Medicina. 2008;22(2-3):69-74.

15. Lunn JN, Farrow SC, Fowkes FGR, Robertson IB, Samuel P. Epidemiology in Anaesthesia I: Anaesthetic Practice Over 20 Years. $\mathrm{Br} J$ Anaesth. 1982;54(8):803.

16. Gareth SA, Kantor FRCPC, Frances CFRCPC. Anaesthesia Drug Cost, Control and Utilization In Canada. Can J Anaesth. 1996;43(1):9-16.

17. WHO guidelines for safe surgery: safe surgery saves lives. (NLM classification: WO 178). 2009 